\title{
Oxygenic Enrichment in Hybrid Ruthenium Sulfide Nanoclusters for Optimized Photothermal Effect
}

Houjuan Zhu, *,t, Zibiao Li, Enyi Ye, ${ }^{+}$David Tai Leong,,,+

${ }^{\dagger}$ Department of Chemical and Biomolecular Engineering, Faculty of Engineering, National University of Singapore, Singapore 117585, Singapore.

Institute of Materials Research and Engineering, A*STAR (Agency for Science, Technology and Research), Singapore 138634, Singapore.

*Corresponding author. Email: cheltwd@nus.edu.sg; zhu_houjuan@imre.a-star.edu.sg
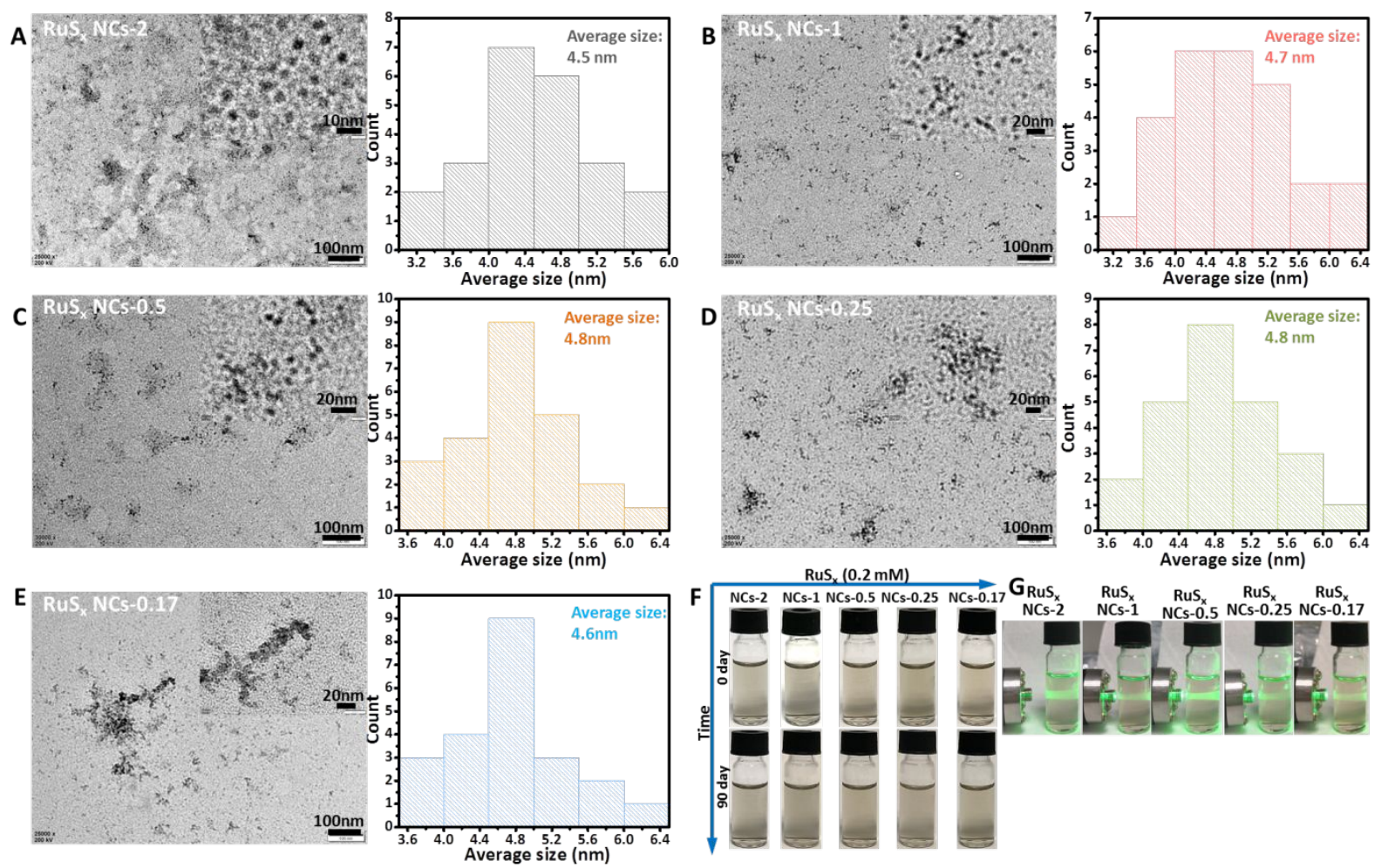
Figure S1. TEM images and corresponding diameter distribution of RuS $\mathrm{x}_{\mathrm{x}} \mathrm{NCs}-2(\mathrm{~A}), \mathrm{RuS}_{\mathrm{x}} \mathrm{NCs}-1$ (B), $\mathrm{RuS}_{\mathrm{x}} \mathrm{NCs}-0.5$ (C), RuS $\mathrm{RCs}_{\mathrm{x}}-2.25$ (D) and RuS $\mathrm{NCs}_{\mathrm{x}} 0.17$ (E); (F) solution color change of RuS $\mathrm{NCs}_{\mathrm{x}}$ before and after storage for 90 days; (G) Tyndall effect of $\mathrm{RuS}_{\mathrm{x}}$ NCs solution.
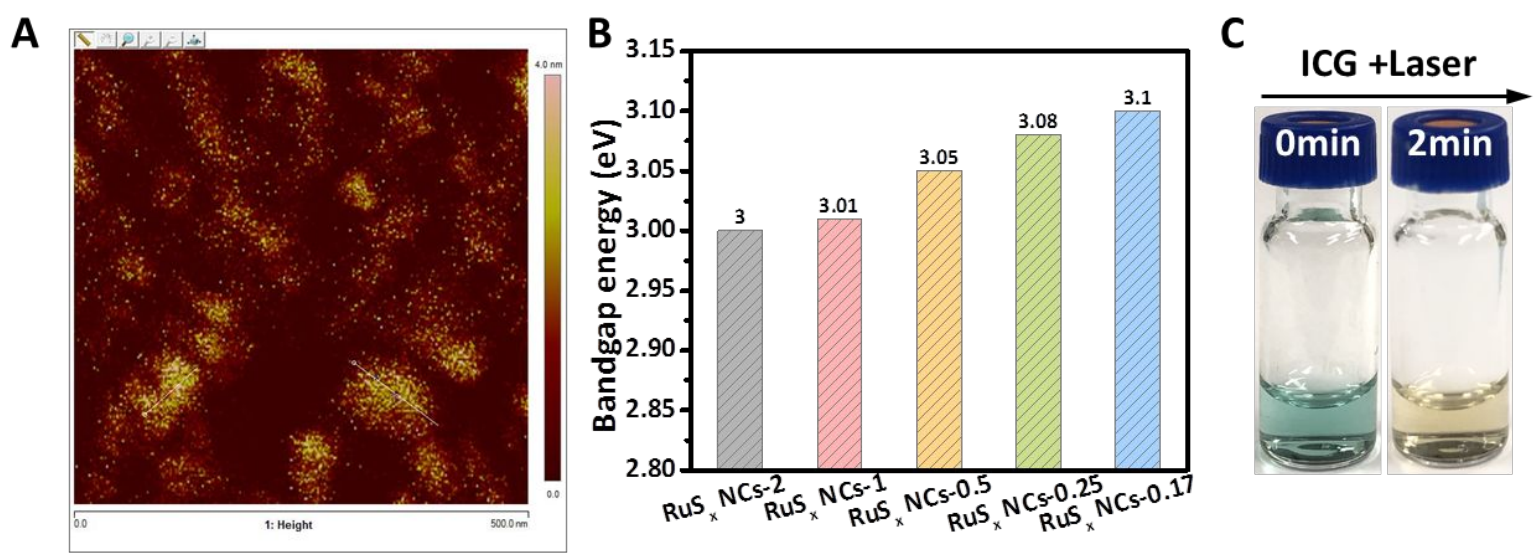

Figure S2. (A) Atomic Force Microscopy (AFM) of RuS $\mathrm{NCs}_{\mathrm{x}}$; ; (B) the bandgap energy of NCs were calculated to be from 3.00 to $3.10 \mathrm{eV}$; (C) solution color of ICG after an $808 \mathrm{~nm}$ laser irradiation with five circles.

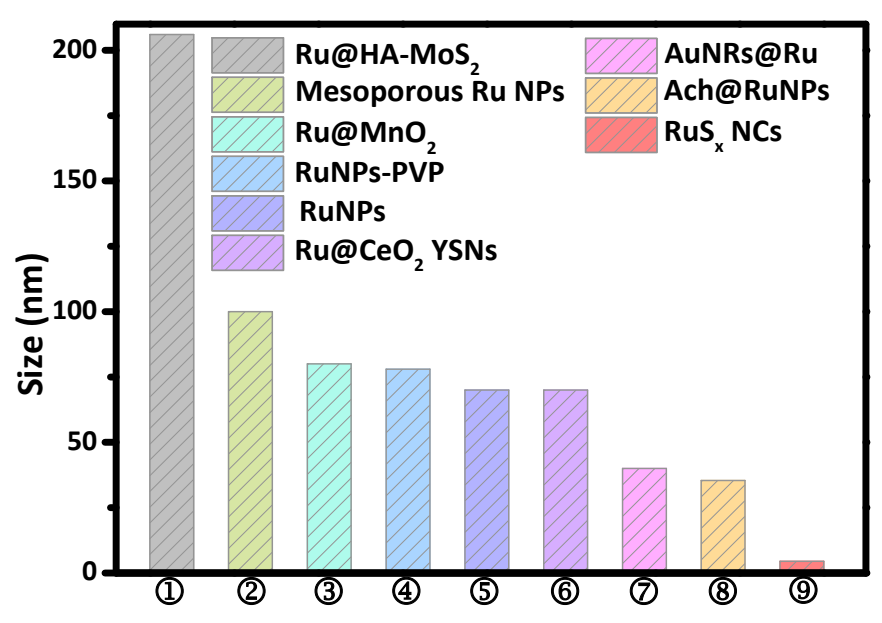

Figure S3. Size of our $\mathrm{RuS}_{\mathrm{x}} \mathrm{NCs}$ in comparison with other $\mathrm{Ru}$ based photothermal nanoagents reported by the references, including Ru@ $\mathrm{HA}-\mathrm{MoS}_{2},{ }^{1}$ mesoporous Ru NPs, ${ }^{1}$ Ru@ MnO $2,{ }^{2}$ RuNPs-PVP, ${ }^{3}$ RuNPs, ${ }^{4} \mathrm{Ru} @ \mathrm{CeO}_{2}$ YSNs,${ }^{5}$ AuNRs@Ru, ${ }^{6}$ Ach@RuNPs ${ }^{3}$. 
Laser +

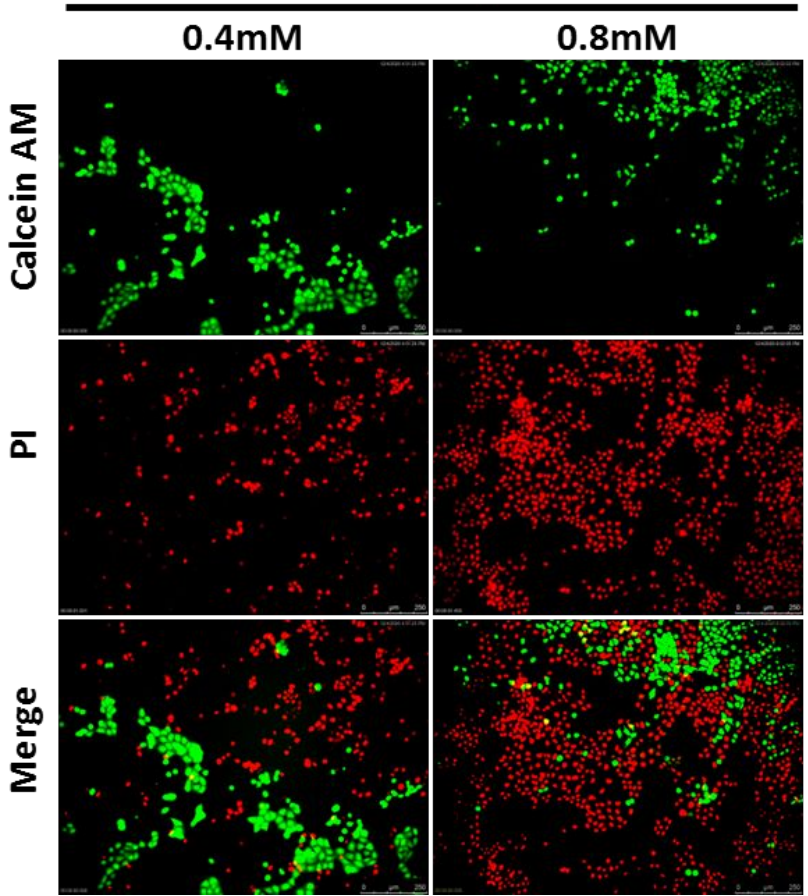

Laser

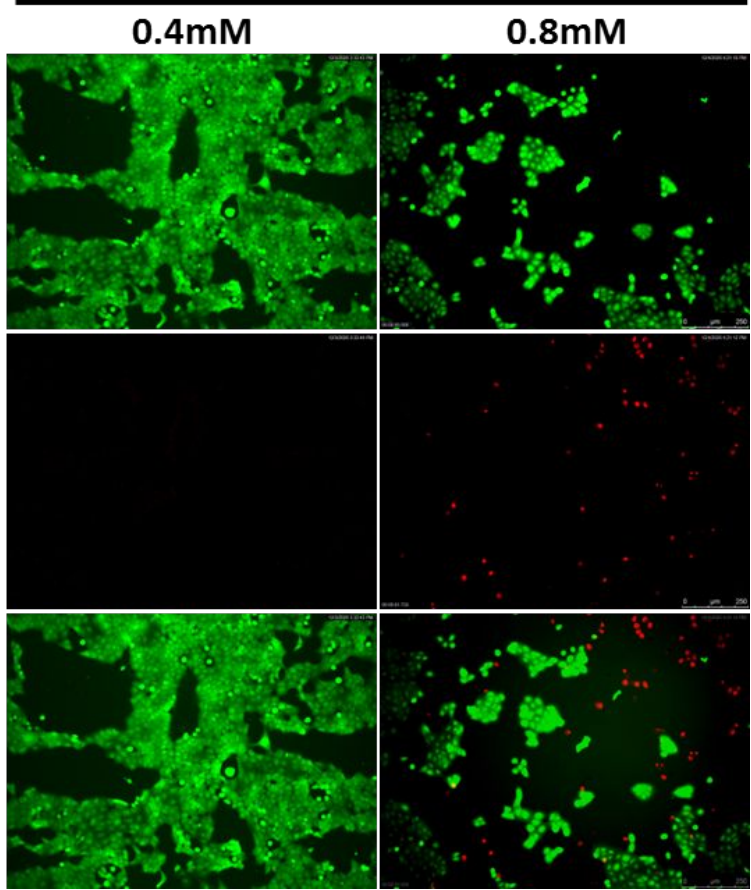

Figure S4. Fluorescence images of Calcein AM/PI co-stained A431 cells incubated with RuS $\mathrm{x}_{\mathrm{x}}$

NCs-1 at the concentration of 0.4 and $0.8 \mathrm{mM}$ with and without NIR laser irradiation for 5 minutes.
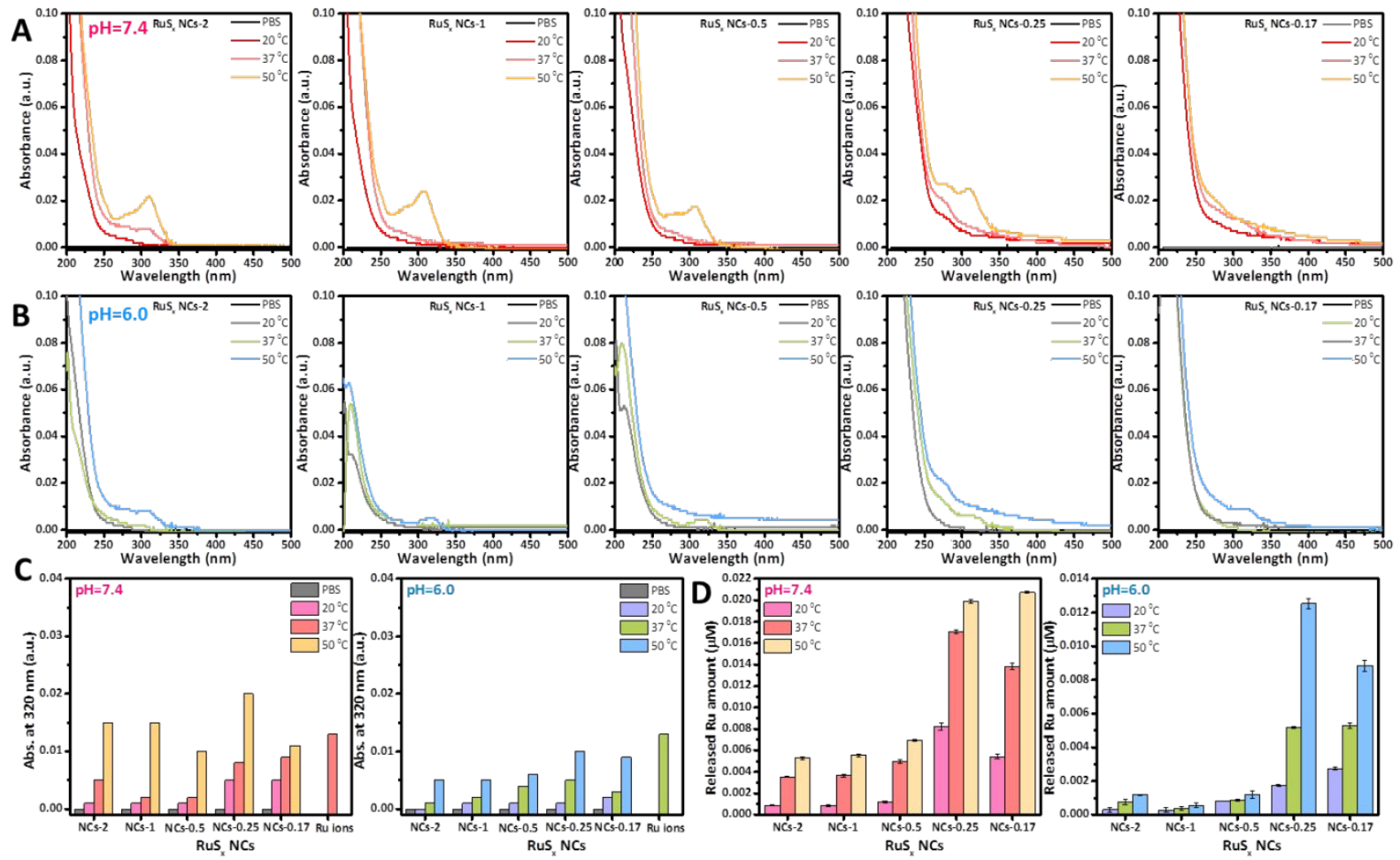

Figure S5. UV-vis spectra of filtered solution after $\mathrm{RuS}_{\mathrm{x}} \mathrm{NCs}$ incubated for 6 days at (A) 
$\mathrm{pH7} .4$ and (B) pH6.0; (C) the absorbance at $320 \mathrm{~nm}$ of $1 \mu \mathrm{M} \mathrm{Ru}{ }^{3+}$ and filtered solution after $\mathrm{RuS}_{\mathrm{x}} \mathrm{NCs}$ incubated in PBS pH7.4 and pH6.0 at $20{ }^{\circ} \mathrm{C}, 30{ }^{\circ} \mathrm{C}, 50{ }^{0} \mathrm{C}$ for 6 days; (D) quantification by ICP-OES of $\mathrm{Ru}^{3+}$ released from $\mathrm{RuS}_{\mathrm{x}} \mathrm{NCs}$ after incubated in PBS pH7.4 and pH6.0 at $20{ }^{\circ} \mathrm{C}, 30{ }^{\circ} \mathrm{C}, 50{ }^{\circ} \mathrm{C}$ for 6 days.

Table S1. The representative peaks of $\mathrm{Ru} 3 \mathrm{p}, \mathrm{Ru} 3 \mathrm{~d}, \mathrm{~S} 2 \mathrm{p}$ and $\mathrm{O} 1 \mathrm{~s}$ for $\mathrm{Ru}, \mathrm{S}$, and $\mathrm{O}$ in the XPS.

\begin{tabular}{|c|c|c|c|c|c|c|}
\hline \multicolumn{2}{|c|}{ XPS peaks } & \multirow{2}{*}{\begin{tabular}{c}
\multicolumn{1}{c}{$\operatorname{RuS}_{\mathrm{x}}$} \\
$\mathrm{NCs}-0.5$ \\
463.4
\end{tabular}} & \multirow{2}{*}{$\begin{array}{r}\operatorname{RuS}_{\mathrm{x}} \text { NCs-1 } \\
462.89\end{array}$} & \multirow{2}{*}{$\begin{array}{r}\mathrm{RuS}_{\mathrm{x}} \text { NCs-2 } \\
463.08\end{array}$} & \multirow{2}{*}{$\begin{array}{r}\mathrm{RuS}_{\mathrm{x}} \text { NCs-4 } \\
462.93\end{array}$} & \multirow{2}{*}{$\begin{array}{r}\text { RuS }_{\mathrm{x}} \text { NCs-6 } \\
462.72\end{array}$} \\
\hline \multirow{4}{*}{$\mathrm{Ru} 3 \mathrm{p}$} & $\mathrm{Ru} 3 \mathrm{p} 3 / 2$ & & & & & \\
\hline & $\mathrm{Ru}^{4+}$ & 466.06 & 466.1 & 466.2 & 466.01 & 466.02 \\
\hline & $\mathrm{Ru} 3 \mathrm{p} 1 / 2$ & 484.93 & 484.7 & 484.88 & 484.61 & 484.65 \\
\hline & $\mathrm{Ru}^{4+}$ & 486.95 & 486.95 & 486.8 & 486.98 & 486.95 \\
\hline \multirow{5}{*}{$\mathrm{Ru} 3 \mathrm{~d}$} & $\mathrm{Ru} 3 \mathrm{~d} 5 / 2$ & 281.79 & 281.84 & 281.79 & 281.6 & 281.42 \\
\hline & $\mathrm{C}-\mathrm{C}$ & 284.36 & 284.5 & 284.62 & 284.66 & 284.44 \\
\hline & $\mathrm{Ru} 3 \mathrm{~d} 3 / 2$ & 285.04 & 285.17 & 284.95 & 285.15 & 284.94 \\
\hline & $\mathrm{Ru}^{6+}$ & 286.27 & 286.34 & 286.3 & 286.42 & 286.21 \\
\hline & $\mathrm{C}=\mathrm{C}$ & 287.86 & 287.85 & 287.96 & 288.01 & 287.89 \\
\hline \multirow{5}{*}{$S 2 p$} & $\mathrm{~S} 2 \mathrm{p} 3 / 2$ & 161.48 & 161.8 & 161.92 & 161.28 & 161.68 \\
\hline & S 2p1/2 & 163.84 & 163.89 & 163.72 & 163.27 & 163.7 \\
\hline & C-S-C & & & 164.64 & 164.4 & 164.89 \\
\hline & S-O & & 166.4 & 166.68 & 166.82 & 166.8 \\
\hline & S-O & 167.5 & 168.56 & 169.37 & 169.36 & 169.67 \\
\hline O 1s & O I & 531.38 & 531.26 & 531.44 & 531.58 & 531.41 \\
\hline
\end{tabular}




\begin{tabular}{|l|l|r|r|r|r|r|}
\hline & O II & 532.84 & 532.72 & 532.68 & 533.04 & 532.92 \\
\hline
\end{tabular}

\section{Reference}

[1] Liu Y., Lin A., Liu J., Chen X., Zhu X., Gong Y., Yuan G., Chen L.; Enzyme-Responsive Mesoporous Ruthenium for Combined Chemo-Photothermal Therapy of Drug-Resistant Bacteria. ACS Appl Mater Interfaces 2019, 11, 26590-26606.

[2] Zhu X., Chen X., Huo D., Cen J., Jia Z., Liu Y., Liu J.; A hybrid nanozymes in situ oxygen supply synergistic photothermal/chemotherapy of cancer management. Biomater Sci 2021, 9, 5330-5343.

[3] Huang X., Chen G., Pan J., Chen X., Huang N., Wang X., Liu J.; Effective PDT/PTT dual-modal phototherapeutic killing of pathogenic bacteria by using ruthenium nanoparticles. J Mater Chem B 2016, 4, 6258-6270.

[4] Zhao S., Zhu X., Cao C., Sun J., Liu J.; Transferrin modified ruthenium nanoparticles with good biocompatibility for photothermal tumor therapy. J Colloid Interface Sci 2018, $511,325-334$.

[5] Zhu X., Gong Y., Liu Y., Yang C., Wu S., Yuan G., Guo X., Liu J., Qin X.; Ru@CeO yolk shell nanozymes: Oxygen supply in situ enhanced dual chemotherapy combined with photothermal therapy for orthotopic/subcutaneous colorectal cancer. Biomaterials 2020, 242, 119923.

[6] Zhang P., Wang J., Huang H., Qiu K., Huang J., Ji L., Chao H.; Enhancing the photothermal stability and photothermal efficacy of AuNRs and AuNTs by grafting with $\mathrm{Ru}(\mathrm{ii})$ complexes. J Mater Chem B 2017, 5, 671-678. 